\title{
Comparison of Dacron ring and suture annuloplasty for aortic valve repair - a porcine study
}

\author{
Leila Louise Benhassen ${ }^{1,2}$, Diana Mathilde Ropcke ${ }^{1,2}$, Mona Sharghbin ${ }^{1,2}$, Troels Lading ${ }^{1,2}$, \\ Jens Kæstel Skov ${ }^{1,2}$, Marcell Juan Tjørnild ${ }^{1,2}$, Karen Bagger Poulsen ${ }^{1,2}$, Tommy Bechsgaard ${ }^{1,2,3}$, \\ Søren Nielsen Skov ${ }^{1,2}$, Sten Lyager Nielsen ${ }^{1,2}$, John Michael Hasenkam ${ }^{1,2}$ \\ ${ }^{1}$ Department of Clinical Medicine, Faculty of Health, Aarhus University, Aarhus, Denmark; ${ }^{2}$ Department of Cardiothoracic and Vascular Surgery, \\ Aarhus University Hospital, Aarhus, Denmark; ${ }^{3}$ Department of Engineering, Faculty of Science and Technology, Aarhus University, Aarhus, Denmark \\ Correspondence to: Leila Louise Benhassen, MD. Department of Cardiothoracic and Vascular Surgery, Aarhus University Hospital, Palle Juul-Jensens \\ Boulevard 99, 8200 Aarhus N, Denmark. Email: benhassen@clin.au.dk.
}

Background: A subvalvular annuloplasty is often used for aortic valve repair in patients with isolated aortic
regurgitation with aortic annulus dilatation. Our aim was to characterize and compare annulus geometry and
dynamics of the Dacron ring and suture annuloplasty and compare it with the native aortic annulus under
standardized conditions. Methods: We randomized 29 pigs of $80 \mathrm{~kg}$ into a Dacron ring group, a suture annuloplasty group and a native control group. The assessment was performed using sonomicrometry crystals for evaluation of dynamic geometry, and pressure measurements and echocardiography to evaluate valve performance.

Results: Aortic annulus area (AAA) was significantly reduced in the Dacron and Suture group compared with the Native group. Expansibility was similar and within normal physiologic limits in all three groups (Native: $12 \% \pm 7 \%$; Dacron: $11 \% \pm 3 \%$; Suture: $10 \% \pm 4 \%$ ). The largest segmental expansion was observed at the right coronary sinus (RC) in the Native and Dacron group but in the Suture group there was no significant difference between segments. The aortic annulus was primarily oval in systole and became more circular in diastole in the Native and Dacron group, however, in the Suture group, the sphericity remained relatively unchanged throughout the cardiac cycle.

Conclusions: This study is the first to describe and compare detailed segmental geometry of the Dacron ring and suture annuloplasty in a standardized porcine model. The two annuloplasties effectively downsized the aortic annulus, while expansibility was maintained. Each annuloplasty had its own geometrical characteristics, but the Dacron ring was more similar to the native aortic annulus than the suture annuloplasty. This study suggests that the Dacron ring offers a more physiological and standardized support by mimicking the geometry and dynamics of the native aortic annulus and thus is a preferable choice over the suture annuloplasty for valve-sparing aortic root procedures.

Keywords: Aortic annulus; valve-sparing procedures; Dacron ring annuloplasty; suture annuloplasty; dynamic characterization; aortic regurgitation

Submitted Jan 31, 2019. Accepted for publication: Apr 11, 2019.

doi: 10.21037/acs.2019.04.02

View this article at: http://dx.doi.org/10.21037/acs.2019.04.02

\section{Introduction}

Valve-sparing techniques are gaining increasing attention as an attractive alternative to composite graft and valve replacement for selected patients with aortic root aneurysms (1). Aortic valve repair with a subvalvular annuloplasty ring is mostly used to treat isolated aortic regurgitation with aortic annulus dilatation or as an adjunct procedure to valve-sparing aortic root procedures (2-7).

The two most commonly used annuloplasty techniques for aortic valve repair is the Dacron ring annuloplasty 
obtained from Dacron tube grafts (Vascutek, Terumo, Japan) and the polytetrafluoroethylene (PTFE) suture annuloplasty. Both annuloplasties have shown good results with or without the remodeling procedure (8-11). However, some concerns have been raised regarding the expansibility of the Dacron ring annuloplasty throughout the cardiac cycle, because the material of the Dacron tube graft is less compliant than the native tissue and is thought to have limited radial expansion capacity. The suture annuloplasty is considered to be more flexible, however, the placement of the suture annuloplasty is less standardized than the ring annuloplasty.

No annuloplasty procedure has been proven superior, and the two most commonly used annuloplasty procedures, the Dacron ring and suture annuloplasty, have never been systematically compared, nor have geometrical changes after annuloplasty implantation been examined in a standardized setting. A complete understanding of the two annuloplasties' impact on native aortic annular geometry and dynamics could potentially give important insight into mechanisms of re-dilatation and surgical failure after aortic valve-sparing procedures with a subvalvular annuloplasty.

Based on these considerations, we hypothesized that the two annuloplasty procedures would downsize the aortic annulus equally, whereas the suture annuloplasty was expected to maintain expansibility throughout the cardiac cycle better than the Dacron ring. Hence, the aim of this porcine study was to characterize and compare:

* The overall expansibility and segmental expansion of the aortic annulus after implantation of the two ring annuloplasties;

* The change in shape of the two ring annuloplasties compared with the native aortic annulus.

\section{Methods}

The study material comprised 29 pigs of $80 \mathrm{~kg}$ (Mixed Duroc and Landrace-Yorkshire). Prior to arrival at the laboratory, each pig was randomized into one of three groups: The Dacron ring group, the Suture annuloplasty group and the Native group serving as controls. The study complied with Danish guidelines for experimental animal research and was approved by the Danish Inspectorate of Animal Experimentation, No. 2013-15-2934-00915.

\section{Experimental protocol}

For geometrical assessment, six $2 \mathrm{~mm}$ sonomicrometry crystals (Sonometrics Corp., London, Ontario, Canada) were implanted at the level of the aortic annulus with one at each nadir of the leaflet and one at each interleaflet triangle to get an anatomically correct segmental description of data (Figure 1A,B). The sonomicrometry method has been extensively validated and has been described in detail in earlier porcine studies $(12,13)$.

\section{Surgical protocol}

Transportation, medication and handling of the animals have previously been described in detail $(14,15)$. The animals were fully anaesthetized during the operation, as previously described. After baseline echocardiography, extra-corporal circulation (ECC) and cardioplegic arrest were established. The aortic valve was exposed through a transverse aortotomy approximately one $\mathrm{cm}$ downstream of the sinotubular junction. The sonomicrometry crystals were inserted through a small incision in the apex of the left ventricle. Each of the six crystals was fixated at each nadir of the leaflet and interleaflet triangle at annular level using a 2-0 PremiCron suture (B. Braun, Melsungen, Germany ${ }^{\circledR}$ ).

A standardized approach was used for the two annuloplasty procedures (Figure 2). For the suture annuloplasty, a CV-0 PTFE double needle suture (GORE-TEX $\mathrm{VR}_{\mathrm{VR}}$, W.L.Gore \& Associates, Flagstaff, AZ, USA) was used and inserted as described by Schneider et al. (3). The suture annuloplasty was placed externally at the level of the aortic annulus and was tied around a $19 \mathrm{~mm}$ Hegar dilator for sizing control.

For the Dacron ring procedure, an open ring of Dacron tube graft with a height of $4 \mathrm{~mm}$ and a diameter of $22 \mathrm{~mm}$ was used for this study. The Dacron ring annuloplasty was anchored with six U-stitches around the aortic annulus and tied down to fasten the ring in the subvalvular position as described by Lansac et al. (7). Due to the close proximity of the left coronary artery to the myocardium in the pig, it was not possible to position the ring under the left main coronary artery, thus the ring annuloplasty was opened and secured closely at each side of the left coronary artery with U-stitches. The aorta was closed using running 4-0 Prolene ${ }^{\circledast}$ sutures. Mikro-Tip pressure catheters (SPR-350, Millar Instruments, Houston, TX, USA) were placed in the left ventricle through the apex and the ascending aorta through the aortotomy. After reperfusion, weaning off ECC and hemodynamic stabilization, data collection was performed for 20 seconds, with collection of simultaneous geometrical-, pressure- and ECG data. Two-dimensional echocardiography (Vivid I, GE Vingmed Ultrasound AS, 

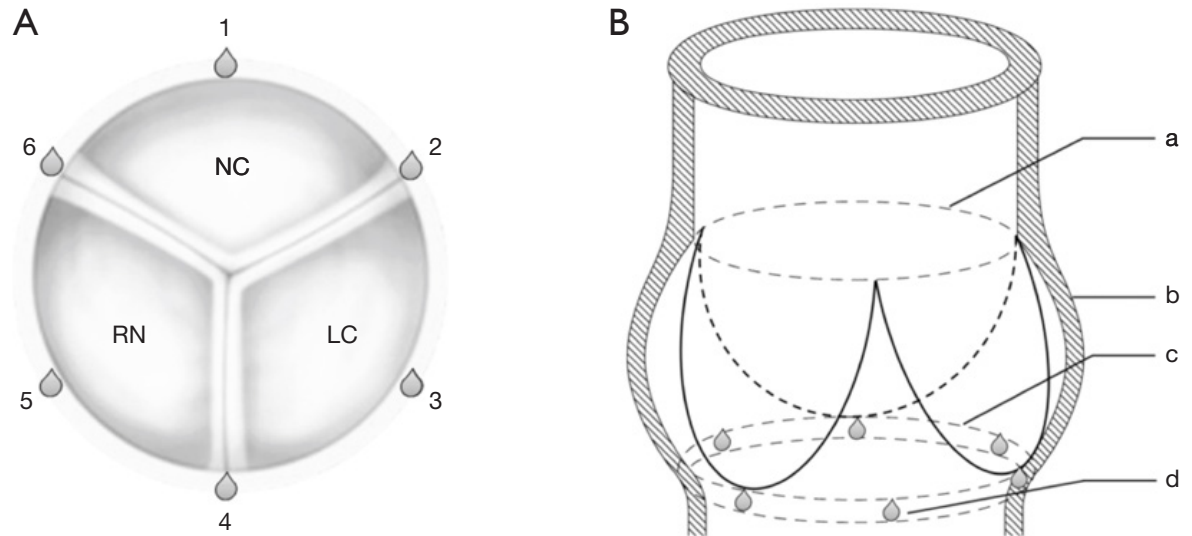

Figure 1 Schematic illustration of the position of the six sonomicrometry crystals at the level of the aortic annulus. (A) The aortic annulus from a cross-sectional view with the sonomicrometry crystals numbered 1-6; (B) the aortic root from a longitudinal view: (a) sinotubular junction; (b) sinuses of Valsalva; (c) aortic annulus; (d) sonomicrometry crystal.
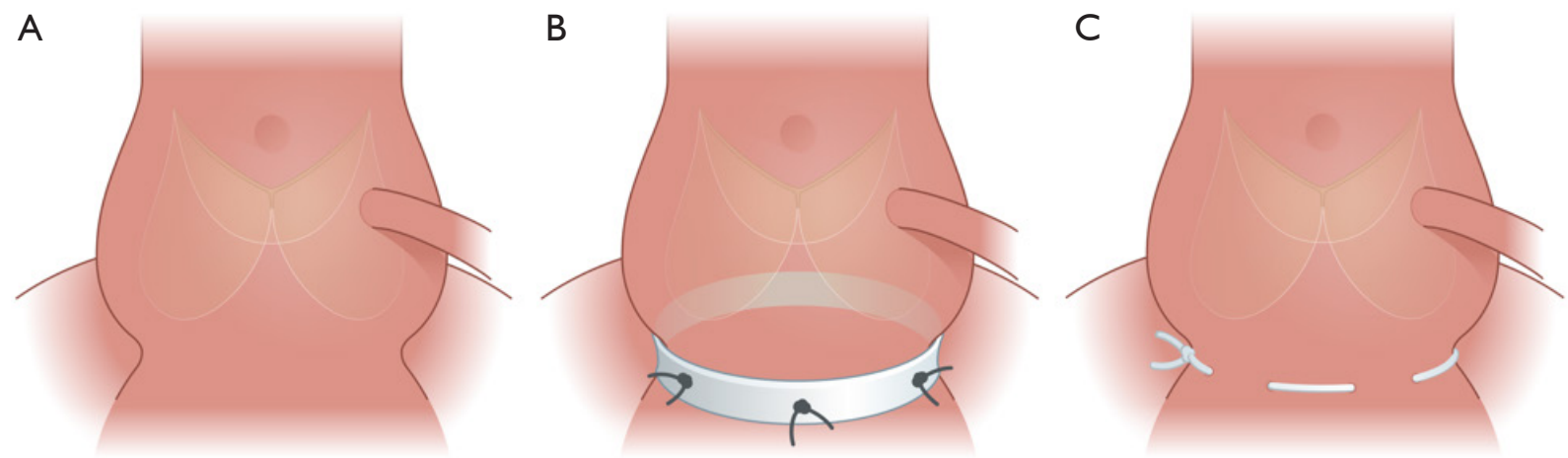

Figure 2 Schematic illustration of the three groups. (A) Native aortic annulus; (B) Dacron ring annuloplasty; (C) suture annuloplasty.

Horten, Norway) was performed to verify valve competence at baseline, and after annuloplasty implantation. The animals were euthanized under continuous anesthesia with intravenous injection of an overdose of pentobarbital. The heart was excised and the position of the sonomicrometry crystals and annuloplasties was verified. All animals were operated under standardized conditions by the same surgeon.

In pilot studies we measured the internal annular diameter of the native aortic annulus with echocardiography to be $23 \mathrm{~mm}$ in a systolic long-axis view. The size of the two annuloplasties was based on the objective to obtain a mild to moderate downsizing of the aortic annulus diameter of $10-15 \%$ in systole. A down-sizing of the aortic annulus of $10-15 \%$ in diameter was compatible with an internal diameter of $19 \mathrm{~mm}$. For the suture annuloplasty, we therefore used a $19 \mathrm{~mm}$ Hegar dilator for sizing control.
For the Dacron annuloplasty we used the following equation to calculate the external ring circumference $(C)$ as described by de Kerchove et al. (16).

$\left(\mathrm{C}(\mathrm{mm})=\left(\mathrm{dia}_{\text {internal }}+4\right) \pi\right)$

Where dia $_{\text {internal }}$ is the internal diameter of $19 \mathrm{~mm}$ and the addendum $4 \mathrm{~mm}$ is the thickness of the aortic wall. Subtracting $4 \mathrm{~mm}$ to the external circumference due to the opening of the ring around the left coronary artery, resulted in a size $22 \mathrm{~mm}$ Dacron ring.

\section{Data acquisition and data analysis}

The left ventricular and aortic pressures were acquired using Mikro-Tip catheters and amplified with a pressure control unit (PCI-2000, Millar Instruments). Sonomicrometry data was collected with a sample rate of $297 \mathrm{~Hz}$ using the Sonometrics TRX USB transceiver system and the 


\begin{tabular}{|c|c|c|c|c|c|c|}
\hline Parameter & Native $(\mathrm{n}=7)$ & Dacron $(\mathrm{n}=9)$ & Suture $(n=7)$ & Native vs. Dacron & Native vs. Suture & Dacron vs. Suture \\
\hline $\mathrm{HR}\left(\min ^{-1}\right)$ & $96 \pm 18$ & $99 \pm 15$ & $98 \pm 18$ & ns & ns & ns \\
\hline LVP $\max (\mathrm{mmHg})$ & $101 \pm 11$ & $88 \pm 8$ & $87 \pm 11$ & $<0.05$ & $<0.05$ & ns \\
\hline Total ECC time (min) & $183 \pm 20$ & $191 \pm 29$ & $200 \pm 26$ & ns & ns & ns \\
\hline Cross clamp time (min) & $114 \pm 13$ & $122 \pm 17$ & $115 \pm 24$ & ns & ns & ns \\
\hline
\end{tabular}

Mean \pm standard deviation. HR, heart rate; LVP, left ventricular pressure; max, maximum; TVP, transvalvular pressure; ns, non-significant.

SonoLabDS3 acquisition software package (Sonometrics Corp.).

The time derivate of the left ventricular pressure (LV-dP/ $\mathrm{dt}$ ) was used for synchronization between the analogue and sonomicrometry signals. The time points were defined as follows:

* Mid-systole: time point between $\mathrm{dP} / \mathrm{dt}$ maximum and minimum;

* Mid-diastole: time point between $\mathrm{dP} / \mathrm{dt}$ minimum and maximum;

* Minimum and maximum time points were those where the parameter reported reached its minimum and maximum value, respectively.

Geometrical values are reported from minimum to maximum (Min-Max). Three segmental distances were used for analysis: the lengths of the non-coronary sinus (NC), right coronary sinus (RC), and the left coronary sinus (LC). For analysis of the shape of the aortic annulus, three anatomical cross-sectional diameters corresponding to the sinus-commissure diameter for each segment throughout the cardiac cycle were calculated: NC-left/right interleaflet triangle (crystal 1-4), RC-left/non-coronary interleaflet triangle (crystal 2-5), LC-right/non-coronary interleaflet triangle (crystal 3-6) (Figure 1). Cross-sectional diameters are reported from mid-diastole to mid-systole (MD-MS). The difference between the smallest and largest crosssectional diameter was calculated and the relative difference between the two was calculated as a measure of the sphericity.

\section{Excluded data}

Five out of 29 included pigs could not be weaned from ECC; two from the Native group, one from the Dacron group and two from the Suture group. This resulted in 24 pigs included for analysis; seven pigs in the Native group, nine pigs in the Dacron group and eight pigs in the Suture group. One pig in the Suture group had several malfunctioning crystals, which resulted in unreliable data and was excluded. Additionally, one pig in the Native group and two pigs from the Suture group had one malfunctioning sonomicrometry crystal, however, those pigs were not excluded from analysis.

\section{Statistical analysis}

All data are presented as mean \pm standard deviation from ten consecutive heart cycles with a significance level of $\mathrm{P}<0.05$. The collected data were analyzed by two-way repeated measures ANOVA using group and repetitive heart cycles as factors. The model allowed for different residual variations in the groups. Groups were then compared using post-boc Wald z-tests. Residuals were inspected for normality and no reason to refute this was found. Heart rate, cross-clamp time and ECC time were compared between groups using a one-way analysis of variance. The data were analyzed using Stata 13.0 (StataCorp LLC, Texas, USA). Development of the statistical models was performed with support from Aarhus University (Biostatistical Advisory Service, University of Aarhus, Aarhus, Denmark).

\section{Results}

\section{Hemodynamic results}

Hemodynamic parameters are presented in Table 1. There was no significant difference between ECC and cross clamp time between groups. The transvalvular pressure loss across the aortic valve did not reveal any significant difference 


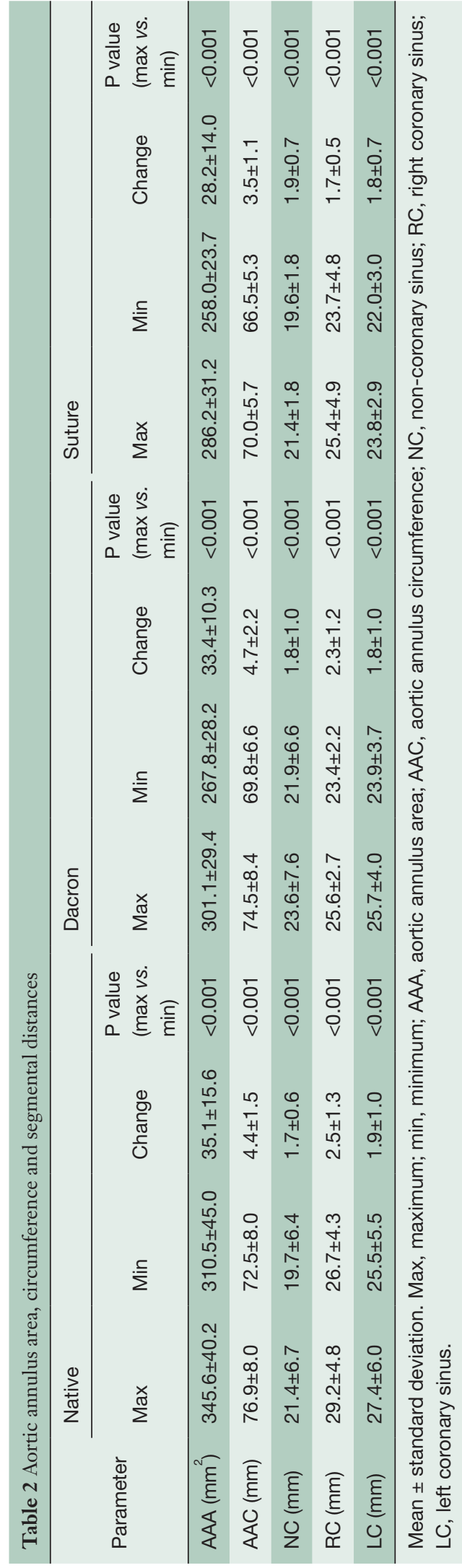

between groups. There was significantly higher left ventricular pressure in the Native group compared to the Dacron and Suture group. All pigs had no or trivial aortic regurgitation on epicardial echocardiography at baselinefollowing annuloplasty implantation, valve function was re-assessed intraoperatively to ensure this status was maintained. The results presented in this manuscript were obtained during the hemodynamic conditions listed in Table 1.

\section{Geometrical results}

Geometrical results are summarized in Tables 2 and 3. Aortic annulus area (AAA) and aortic annulus circumference (AAC) are presented in Figure 3 at four defined time points throughout the cardiac cycle for each group. Both the Dacron and Suture group had a significantly reduced AAA compared with the Native group resulting in a reduction of $13 \%$ in the Dacron group and $17 \%$ in the Suture group. We found no difference in AAA between the Dacron and Suture group. Expansibility was not statistically different between groups (Native: $12 \% \pm 7 \%$; Dacron: $11 \% \pm 3 \%$; Suture: $10 \% \pm 4 \%$ ), which was all within the normal physiological limit (6). There was also a significant difference between AAC within each group, with an expansion of $6 \% \pm 2 \%$ in the Native group, $6 \% \pm 2 \%$ in the Dacron group and $5 \% \pm 2 \%$ in the Suture group.

Segmental expansion from Min-Max for each of the three annular segments in each group is summarized in Table 2. In the Native and Dacron group, the largest annular expansion was observed at the RC and the smallest at the NC from Min-Max (Table 2). In the Suture group, there was no difference in segmental expansion in any of the three segments of the annulus.

The cross-sectional diameters are presented in Figure 4 at four defined time points throughout the cardiac cycle and summarized in Table 3 for all three groups from MD-MS. In the Native and the Dacron group, the largest and smallest cross-sectional diameter was observed for diameter 1-4 and $2-5$, respectively at both mid-systole and mid-diastole in both groups. In the Suture group, the largest cross-sectional diameter was similarly diameter $1-4$, however, there was no significant difference between diameter 2-5 and 3-6. In all three groups, there was a significantly larger expansion for diameter 1-4 compared with the two other cross-sectional diameters within each group, with a mean expansion of $1.5 \pm 0.9 \mathrm{~mm}$ in the Native group, $1.9 \pm 0.6 \mathrm{~mm}$ in the Dacron group, and $1.1 \pm 0.6 \mathrm{~mm}$ in the Suture group. The 


\begin{tabular}{|c|c|c|c|c|c|c|}
\hline Parameter & Native & Dacron & Suture & Native vs. Dacron & Native vs. Suture & Dacron vs. Suture \\
\hline \multicolumn{7}{|l|}{ Crystal 1-4 } \\
\hline $\mathrm{MS}(\mathrm{mm})$ & $26.5 \pm 1.0$ & $24.1 \pm 1.7$ & $24.1 \pm 1.0$ & $<0.05$ & $<0.05$ & ns \\
\hline Change (mm) & $1.5 \pm 0.9$ & $1.9 \pm 0.6$ & $1.1 \pm 0.6$ & ns & ns & $<0.05$ \\
\hline \multicolumn{7}{|l|}{ Crystal 2-5 } \\
\hline $\mathrm{MS}(\mathrm{mm})$ & $20.1 \pm 2.9$ & $17.2 \pm 1.7$ & $18.7 \pm 1.5$ & $<0.05$ & ns & ns \\
\hline $\mathrm{MD}(\mathrm{mm})$ & $20.5 \pm 2.8$ & $17.7 \pm 1.7$ & $18.2 \pm 2.2$ & $<0.05$ & $<0.05$ & ns \\
\hline \multicolumn{7}{|l|}{ Crystal 3-6 } \\
\hline MS (mm) & $22.9 \pm 1.3$ & $20.4 \pm 2.6$ & $18.5 \pm 1.8$ & $<0.05$ & $<0.05$ & ns \\
\hline $\mathrm{MD}(\mathrm{mm})$ & $22.4 \pm 1.5$ & $20.1 \pm 2.9$ & $19.4 \pm 2.3$ & ns & $<0.05$ & ns \\
\hline Change (mm) & $0.5 \pm 1.0$ & $0.2 \pm 0.5$ & $-0.8 \pm 0.6$ & ns & $<0.05$ & $<0.05$ \\
\hline
\end{tabular}

Mean \pm standard deviation. MS, mid-systole; MD, mid-diastole; ns, non-significant. Crystals 1-6 representing sonomicrometry crystals.

diameter was downsized on average $11 \%$ (range, $9-15 \%$ ) in the Dacron group and 12\% (range, 7-19\%) in the Suture group compared with the Native group throughout the cardiac cycle. We found that the aortic annulus was oval at mid-systole and became more circular at Mid-diastole in the Native and Dacron group, with a relative difference between the two cross-sectional diameters of $36 \%$ in midsystole and $25 \%$ in mid-diastole in the Native group and $42 \%$ in mid-systole and $29 \%$ in mid-diastole in the Dacron group. However, in the Suture group, the sphericity remained relatively unchanged throughout the cardiac cycle with a relative difference between cross-sectional diameters of $38 \%$ in mid-systole and $36 \%$ in mid-diastole.

\section{Discussion}

In this acute porcine study, we evaluated the dynamics of the aortic annulus after implantation of the Dacron ring and suture annuloplasty with segmental geometrical measurements. The model was successful for the intended characterization with sonomicrometry crystals to measure geometrical changes throughout the cardiac cycle. This study is the first to systematically compare the Dacron ring to the suture annuloplasty under standardized conditions with detailed geometrical measurements in an in vivo study.
Both annuloplasty procedures downsized the annulus diameter $11-12 \%$, which was in accordance with our aim of downsizing $10-15 \%$. We found a preserved expansibility with no difference between groups. From our previous in vitro study (17), annular distensibility was also found to be preserved in all three groups assessed by 2D echocardiography. Basmadjian et al. (6) evaluated the expansibility of the aortic annulus by 2D echocardiography and found that the expansibility of the aortic annulus was preserved after implantation of the Dacron ring, even up to two years postoperatively with an expansibility of $10 \%$. The authors concluded that it cannot be determined by $2 \mathrm{D}$ echocardiography whether the measured change in systolic dimension is a systolic expansion or it is merely a change in spatial conformation of the annulus. Lansac et al. (9) found annular expansibility to be preserved in patients with a Dacron ring, however, the authors also concluded that this might be explained by the change in shape of the annulus. From our study, we can conclude with certainty that even though there is indeed a change in spatial conformation throughout the cardiac cycle, there is also a significant increase in annular dimensions from diastole to systole, which indicates that both the Dacron ring and the suture annuloplasty have deformational potential.

We found that there was a change in shape of the aortic 



Figure 3 Aortic annulus area (AAA) (left) and aortic annulus circumference (AAC) (right) presented at four defined time points throughout the cardiac cycle (ED, MS, ES, MD). Mean + standard deviation. (A) Native group; (B) Dacron group; (C) Suture group. ED, end-diastole; MS, mid-systole; ES, end-systole; MD, middiastole.

annulus throughout the cardiac cycle in the Native and Dacron group, and that the annulus was oval in systole and became more circular in diastole. Few studies have investigated the shape of the aortic annulus in patients by CT and 2D echocardiography; Suchá et al. (18) investigated the aortic annulus from two perpendicular cross-sectional diameters that were not anatomically defined. They found that the short diameter axis changed

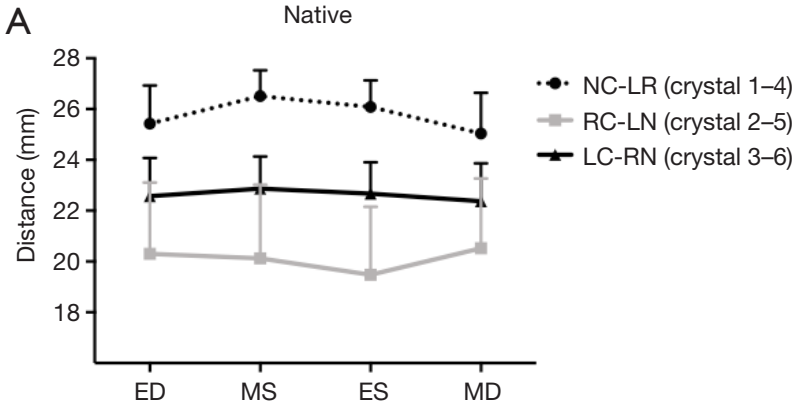

B Dacron



C

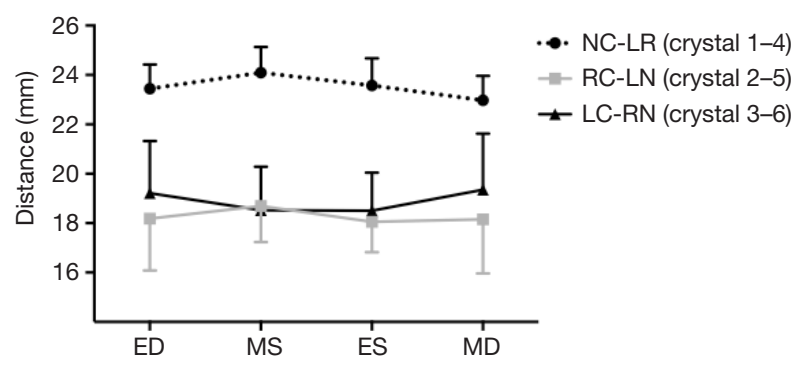

Figure 4 Cross-sectional diameters NC-LR, RC-LN and LC-RN presented at four defined time points throughout the cardiac cycle (ED, MS, ES, MD). Mean \pm standard deviation. (A) Native group; (B) Dacron group; (C) Suture group. ED, end-diastole; MS, midsystole; ES, end-systole; MD, mid-diastole; NC, non-coronary sinus; LR, left/right interleaflet triangle; RC, right coronary sinus; $\mathrm{LN}$, left/non-coronary interleaflet triangle; LC, left coronary sinus; RN, right/non-coronary interleaflet triangle.

significantly in dimension, whereas the maximum diameter remained relatively unchanged, resulting in an oval shaped annulus in diastole and circular shaped annulus in systole. However, their definition of sphericity was not specified, the evaluation of the aortic annulus was based on both echocardiography and CT scan and the patient population consisted of both healthy adults and patients with aortic valve stenosis. de Heer et al. (19) investigated the 
deformation of the aortic annulus in healthy adult patients and concluded that the aortic annulus was oval in both systole and diastole in most patients, which is in accordance with our results.

The Dacron group had similar geometrical results compared with the Native group, but the Suture group differed in both geometry and dynamics. A possible explanation could be that the Dacron ring is fixed with six anchoring U-stitches at the subvalvular plane, which divides the annulus into six compartments, leaving only the space between the sutures to expand (6). If one section of the aortic annulus expands, the expansion cannot pass on to other compartments because of the anchoring sutures. In contrast, the suture annuloplasty is not fixed at the level of the aortic root, but is only passed through the tissue externally at the level of the aortic annulus. If one section of the aortic annulus expands, the expansion should be able to pass on to any section of the circumference, since they communicate. A drawback of the Dacron-ring is the need for extensive external dissection to reach the plane of the aortic annulus, increasing the risk of coronary artery and right ventricular perforation $(20,21)$, which is not needed for implantation of the suture annuloplasty. However, implantation of the Dacron ring is considered to be a more standardized procedure with anchoring sutures at six defined anatomical locations ensuring that the Dacron ring is implanted in a consistent manner. The suture annuloplasty showed a remodeling effect of the native aortic annulus and is less predictable in what way it affects the movement and physiology of the native aortic annulus. The comparability between the Dacron ring and the native aortic annulus suggests that the Dacron ring mimics the movement and physiology of the native aortic annulus better than the suture annuloplasty, which is preferable when performing aortic valve and root repair.

\section{Study limitations}

This study was performed in porcine hearts, which have some differences compared to human hearts; the right coronary sinus of pig hearts has a wide septal muscle shelf just downstream of the insertion of the right cusp, which could potentially change the geometry in the aortic annulus. However, the porcine model is an acknowledged and widely used model with low interindividual variation, which makes it possible to evaluate surgical procedures. Due to the close proximity of the left coronary artery to the myocardium in the pig, the Dacron ring was not closed, but secured at each side of the left main coronary artery. Our study is an acute invasive study, and therefore we cannot draw conclusions on the long-term effects of an annuloplasty ring or whether the preserved expansibility will remain over time. Nonetheless, the annuloplasties' effect on geometry of the aortic annulus and the preserved expansibility after implantation of the two types of annuloplasty rings is a new insight and should be confirmed in long-term human studies.

\section{Conclusions}

From this porcine model, a detailed analysis of the segmental dynamic geometry of the aortic annulus after implantation of the Dacron ring and the suture annuloplasty throughout the cardiac cycle was obtained. Expansibility was preserved similarly with both the Dacron ring and the suture annuloplasty. Both types of annuloplasties effectively reduced the aortic annulus and preserved the dynamics, but the Dacron ring allowed dynamics more comparable to the native aortic annulus. Thus, the Dacron ring might be a preferable choice over the suture annuloplasty for valvesparing aortic root procedures.

\section{Acknowledgments}

Funding: This work was supported by Aarhus University, the Danish Heart Foundation Grant number 16-R107-A6771-22001; the Lundbeck Foundation (grant number R184-2014-2478); the Raimond og Dagmar Ringgård-Bohns Fond til støtte for forskning i hjerteog kræftsygdomme; the Fonden til Lægevidenskabens Fremme Fond; the Helge Peetz og Verner Peetz og hustru Vilma Peetz Legat Fond; the Købmand Sven Hansen og Hustru Ina Hansens Fond; the Ingeniør August Frederik Wedell Erichsens Legat Fond; and the Handelsgartner Ove William Buhl Olesen og ægtefælle fru Edith Buhl Olesens Mindelegat Fond.

\section{Footnote}

Conflicts of Interest: The authors have no conflicts of interest to declare.

\section{References}

1. David TE. Aortic root aneurysms: remodeling or composite replacement? Ann Thorac Surg 1997;64:1564-8.

2. Lansac E, Centa ID, Crozat EA, et al. An external open 
ring for isolated aortic valve repair. Multimed Man Cardiothorac Surg 2011;2011:mmcts.2009.004119.

3. Schneider U, Aicher D, Miura Y, et al. Suture annuloplasty in aortic valve repair. Ann Thorac Surg 2016;101:783-5.

4. Lansac E, Centa ID, Bonnet N, et al. Aortic prosthetic ring annuloplasty: a useful adjunct to a standardized aortic valve-sparing procedure? Eur J Cardiothorac Surg 2006;29:537-44.

5. Lansac E, Di Centa I, Sleilaty G, et al. Remodeling root repair with an external aortic ring annuloplasty. J Thorac Cardiovasc Surg 2017;153:1033-42.

6. Basmadjian L, Basmadjian AJ, Stevens LM, et al. Early results of extra-aortic annuloplasty ring implantation on aortic annular dimensions. J Thorac Cardiovasc Surg 2016;151:1280-5.e1.

7. Lansac E, Di Centa I, Varnous S, et al. External aortic annuloplasty ring for valve-sparing procedures. Ann Thorac Surg 2005;79:356-8.

8. Aicher D, Schneider U, Schmied W, et al. Early results with annular support in reconstruction of the bicuspid aortic valve. J Thorac Cardiovasc Surg 2013;145:S30-4.

9. Lansac E, Di Centa I, Sleilaty G, et al. Long-term results of external aortic ring annuloplasty for aortic valve repair. Eur J Cardiothorac Surg 2016;50:350-60.

10. Lansac E, Di Centa I, Vojacek J, et al. Valve sparing root replacement: the remodeling technique with external ring annuloplasty. Ann Cardiothorac Surg 2013;2:117-23.

11. Holubec T, Higashigaito K, Belobradek Z, et al. An expansible aortic ring in aortic root remodeling: exact position, pulsatility, effectiveness, and stability in threedimensional CT study. Ann Thorac Surg 2017;103:83-90.

12. Lansac E, Lim HS, Shomura Y, et al. A four-dimensional study of the aortic root dynamics. Eur J Cardiothorac Surg 2002;22:497-503.

Cite this article as: Benhassen LL, Ropcke DM, Sharghbin M, Lading T, Skov JK, Tjørnild MJ, Poulsen KB, Bechsgaard T, Skov SN, Nielsen SL, Hasenkam JM. Comparison of Dacron ring and suture annuloplasty for aortic valve repair-a porcine study. Ann Cardiothorac Surg 2019;8(3):342-350. doi: 10.21037/acs.2019.04.02
13. Bechsgaard T, Lindskow $T$, Lading $T$, et al. Biomechanical characterization of the native porcine aortic root. J Biomech 2018;74:156-62.

14. Nielsen SL, Lomholt M, Johansen P, et al. Mitral ring annuloplasty relieves tension of the secondary but not primary chordae tendineae in the anterior mitral leaflet. J Thorac Cardiovasc Surg 2011;141:732-7.

15. Ropcke DM, Ilkjaer C, Skov SN, et al. Functional and Biomechanical Performance of Stentless Extracellular Matrix Tricuspid Tube Graft: An Acute Experimental Porcine Evaluation. Ann Thorac Surg 2016;101:125-32.

16. de Kerchove L, Vismara R, Mangini A, et al. In vitro comparison of three techniques for ventriculoaortic junction annuloplasty. Eur J Cardiothorac Surg 2012;41:1117-23; discussion 1123-4.

17. Sharghbin M, Benhassen LL, Lading T, et al. Comparison of the Dacron ring and suture annuloplasty for aortic root repair: an in vitro evaluation. Interact Cardiovasc Thorac Surg 2018;27:819-27.

18. Suchá D, Tuncay V, Prakken NH, et al. Does the aortic annulus undergo conformational change throughout the cardiac cycle? A systematic review. Eur Heart J Cardiovasc Imaging 2015;16:1307-17.

19. de Heer LM, Budde RP, Mali WP, et al. Aortic root dimension changes during systole and diastole: evaluation with ECG-gated multidetector row computed tomography. Int J Cardiovasc Imaging 2011;27:1195-204.

20. Khelil N, Sleilaty G, Palladino M, et al. Surgical anatomy of the aortic annulus: landmarks for external annuloplasty in aortic valve repair. Ann Thorac Surg 2015;99:1220-6.

21. David TE, Maganti M, Armstrong S. Aortic root aneurysm: principles of repair and long-term follow-up. J Thorac Cardiovasc Surg 2010;140:S14-9. 